\title{
PROBABILISTIC TREATMENT OF THE BLOWING UP OF SOLUTIONS FOR A NONLINEAR INTEGRAL EQUATION
}

\author{
BY \\ MASAO NAGASAWA AND TUNEKITI SIRAO( $\left.{ }^{1}\right)$
}

1. Introduction. The blowing up of the solutions of the following semilinear parabolic equation

$$
\begin{aligned}
\partial u(t, x) / \partial t & =G u(t, x)+c(x) u(t, x)^{\beta}, \quad(\beta \geqq 2) \\
u(0, x) & =f(x), \quad x \in R^{d},
\end{aligned}
$$

depends on the dimension $d$ and power $\beta$, where $\boldsymbol{G}$ is the infinitesimal generator of a linear nonnegative contraction semigroup on the space $B\left(R^{d}\right)$ of bounded measurable functions on $R^{d}$ and $c$ is a bounded nonnegative measurable function on $R^{d}$. This fact was recently proved by Fujita [2] when $G$ is the Laplacian operator. In this paper we will give upper and lower bounds for the solution of (1.1) constructed by a probabilistic method (cf. (3.4) and (4.7)). As a corollary we shall obtain Fujita's result when $\boldsymbol{G}$ is a fractional power $-(-\Delta)^{\alpha}, 0<\alpha \leqq 2$, of the Laplacian operator.

Our method is based on probabilistic arguments relating to the branching Markov processes (cf. Ikeda-Nagasawa-Watanabe [3], Sirao [8] and Nagasawa [7]). The necessary facts of probabilistic arguments in this context will be summarized in $\$ 2$, while in $\$ 3$ and $\$ 4$ we shall give upper and lower bounds of the probabilistic solution of (1.1) and some applications.

2. Preliminaries. Let $D$ be a compact Hausdorff space with a countable open base, $\boldsymbol{B}(D)$ be the space of bounded Borel measurable functions on $D . B^{+}(D)$ denotes the set of nonnegative elements of $B(D)$. Let $\left\{T_{t} ; t \geqq 0\right\}$ be a nonnegative contraction semigroup on $\boldsymbol{B}(D)$ defined through a kernel $T_{t}(x, d y)$ such that

(i) $T_{t}(x, \cdot)$ is a nonnegative Borel measure on $D$ with $T_{t}(x, D) \leqq 1$;

(ii) $T .(\cdot, B)$ is measurable on $[0, \infty) \times D$ for any Borel subset $B$ of $D$,

(iii) $T_{t+s}(x, B)=\int T_{t}(x, d y) T_{s}(y, B)$ for any $t, s \geqq 0, x \in D$ and Borel subset $B$, and

(iv) $T_{t} f(x)=\int T_{t}(x, d y) f(y)$ for $f \in \boldsymbol{B}(D)$.

We shall consider the following nonlinear integral equation with an initial data $f \in \boldsymbol{B}^{+}(D)$ instead of (1.1):

$$
v(t, x)=T_{t} f(x)+\int_{0}^{t} d s T_{s}\left(c \cdot v(t-s, \cdot)^{\beta}\right)(x)
$$

Received by the editors April 1, 1968.

(') The first author was supported by NSF Grant GP 4867 through Cornell University. 
where $c \in \boldsymbol{B}^{+}(D)$, which will be fixed throughout the paper, and $\beta=2,3,4, \ldots$ One may apply the usual successive approximation method to obtain a solution of (2.1). This is, however, not appropriate for our present purpose. We shall treat the nonlinear integral equation in a different way, finding a linear integral equation which is a linear dilatation of the equation (2.1). This linear integral equation will be defined on an enlarged space

$$
S=\bigcup_{n=1}^{\infty} D^{n}
$$

where $D^{n}$ is the symmetric $n$-fold product $\left({ }^{2}\right)$ of $D, n \geqq 1$.

For $f \in B^{+}(D)$, set

$$
\hat{f}(x)=\prod_{j=1}^{n} f\left(x_{j}\right), \quad \text { when } x=\left(x_{1}, x_{2}, \ldots, x_{n}\right) \in D^{n},
$$

$\hat{f}$ is, then, a measurable function on $S$ and $\hat{f} \in B^{+}(S)$ when $f \leqq 1$.

We shall state some fundamental facts which will play an important role in the following discussion.

[a.1] There exist unique nonnegative kernels $T_{t}(x, d y)$ and $\Psi(x, d s d y)$ defined on $[0, \infty) \times S \times S$ and $S \times[0, \infty) \times S$ respectively, such that when $x=\left(x_{1}, x_{2}, \ldots, x_{n}\right)$ $\in D^{n}$

$$
\int_{S} T_{t}(x, d y) \hat{f}(y)=\prod_{j=1}^{n} T_{t} f\left(x_{j}\right), \quad f \in B^{+}(D)
$$

and $\left({ }^{3}\right)$

$$
\int_{s} \Psi(x, d s d y) \hat{f}(s, y)=d s \sum_{k=1}^{n} T_{s}\left(c \cdot f(s, \cdot)^{\beta}\right)\left(x_{k}\right) \prod_{i \neq k: i=1}^{n} T_{s}(f(s, \cdot))\left(x_{i}\right) .
$$

Moreover the support of $\boldsymbol{T}_{t}(\boldsymbol{x}, \cdot)$ is concentrated on $D^{n}$ and that of $\Psi(x, d s \cdot)$ on $D^{n+\beta-1}$ (cf. Ikeda-Nagasawa-Watanabe [3, Lemma 0.3]).

Then we define a linear integral equation with an initial data $\hat{f}$

$$
u(t, x)=T_{t} \hat{f}(x)+\int_{0}^{t} \int_{S} \Psi(x, d s d y) u(t-s, y), \quad x \in S, \quad f \in B^{+}(S),
$$

where

$$
T_{t} \hat{f}(x)=\int_{S} T_{t}(x, d y) \hat{f}(y)
$$

Now set

$$
\begin{aligned}
& u_{0}(t, x)=T_{t} \hat{f}(\boldsymbol{x}) \\
& u_{k}(t, x)=\int_{0}^{t} \int_{S} \Psi(x, d s d y) u_{k-1}(t-s, y), \quad k \geqq 1
\end{aligned}
$$

$\left({ }^{2}\right)$ That is, $D^{n}$ is the quotient space of the $n$-fold product of $D$ by the permutation of the coordinate.

$\left({ }^{3}\right) f(s, x)$ is obtained by applying $(2.3)$ to $f(s, x)$ for fixed $s$. 
[a.2] $u_{k}(t, x)$ is well defined and $\sum_{k=0}^{\infty} u_{k}(t, x)$ converges for sufficiently small $t>0$. If we put

$$
u(t, x)=\sum_{k=0}^{\infty} u_{k}(t, x)
$$

when the right-hand side converges, then it is the minimal (local) solution of (2.6) (cf. [3, Chapter IV]).

[a.3] The most important property of the $u(t, x)$ is the following branching property:

$$
u(t, x)=\prod_{j=1}^{n} u\left(t, x_{j}\right), \quad \text { when } x=\left(x_{1}, x_{2}, \ldots, x_{n}\right) \in D^{n},
$$

(cf. [3, Chapter I]).

[a.4] Accordingly, by the branching property, (2.4), and (2.5), it is easy to see that the restriction of $u(t, x)$ on $D$ is a solution of the nonlinear integral equation (2.1). Moreover, it is the minimal solution of (2.1), since if $v(t, x)$ is a solution of (2.1) then $v(t, x)=\prod_{j=1}^{n} v\left(t, x_{j}\right), x \in D^{n}$ is a solution of (2.6) (cf. [3, Theorem 4.7]). We shall call this minimal solution $u(t, x), x \in D$, of (2.1) obtained through (2.6) the probabilistic solution of $(2.1)\left({ }^{4}\right)$.

[a.5] Let $f_{k_{1}}(s, x)(i=1,2, \ldots, m)$ be in $B^{+}([0, \infty) \times D)$ and $a_{k_{1} \cdots k_{n}}$ be certain constants which are symmetric with respect to $\left(k_{1}, k_{2}, \ldots, k_{m}\right)$. When $\boldsymbol{x}=$ $\left(x_{1}, x_{2}, \ldots, x_{n}\right) \in D^{n}$,

$$
\begin{aligned}
& \int_{D^{m}} \Psi(x, d s d y)\left\{\sum^{(k, m)} a_{k_{1} k_{2} \cdots k_{m}} \prod_{i=1}^{m} f_{k_{i}}\left(s, y_{i}\right)\right\} \\
& =d s \sum_{i=1}^{n} \sum^{(k, m)} a_{k_{1} k_{2} \cdots k_{m}} T_{s}\left(c \cdot \prod^{\beta} f_{k_{i}}(s, \cdot)\right)\left(x_{l}\right) \prod_{j \neq l ; j=1}^{n} T_{s}\left(f_{k}(s, \cdot)\right)\left(x_{j}\right),
\end{aligned}
$$

where $m=n+\beta-1, \sum^{(k, m)}$ denotes the sum over all $\left(k_{1}, k_{2}, \ldots, k_{m}\right)$ satisfying $\sum_{i=1}^{m} k_{i}=k$, and $\prod^{\beta}$ the product over $i=l, n+1, n+2, \ldots, m$. This representation of $\Psi$ follows from the fact that the integrand of the left-hand side of (2.10) can be expressed by a linear combination of functions of the form $\hat{g}, g \in B^{+}(D)$.

We will give upper and lower bounds of $u_{k}(t, x)$ in the following sections.

3. Case 1. There exists a global solution. Now we give an upper bound of $u_{k}(t, \boldsymbol{x})$.

Lemma 3.1. For $f \in \boldsymbol{B}^{+}(D)$ and $\boldsymbol{x}=\left(x_{1}, x_{2}, \ldots, x_{n}\right) \in D^{n}, u_{k}(t, x)$ which is defined by (2.7) has an upper bound

$\left(^{4}\right)$ When $T_{t}$ is the semigroup of $\exp \left(-\int_{0}^{t} c\left(x_{s}\right) d s\right)$-subprocess of a conservative Markov process on $D, u(t, x)$ in (2.8) exists for all $t \geqq 0$ and $U_{t} f(x)=u(t, x)$, where $U_{t}$ is the semigroup of a Markov process on $S$ which has the branching property (branching Markov process). This remark is also true for any $T_{t}$, but we need some additional structure for branching Markov processes (cf. Sirao [8], Nagasawa [7]). 


$$
u_{k}(t, x) \leqq\|c\|^{k} \cdot \frac{\prod_{i=0}^{k-1}\{n+i(\beta-1)\}}{k !} \cdot\left\{\int_{0}^{t} \sup _{y \in D} h(s, y)^{\beta-1} d s\right\}^{k} \cdot \prod_{j=1}^{n} h\left(t, x_{j}\right),
$$

where $k=1,2,3, \ldots$ and

$$
h(t, x)=T_{t} f(x) .
$$

Proof. We shall prove $(3.1)$ by induction. $u_{1}(t, x)$ is estimated as follows: By (2.5) and (2.7)

$$
\begin{aligned}
u_{1}(t, x) & =\int_{0}^{t} d s \sum_{l=1}^{n} T_{s}\left(c \cdot h_{t-s}^{\beta}\right)\left(x_{l}\right) \cdot \prod_{i \neq l} T_{s} h_{t-s}\left(x_{i}\right) \\
& \leqq\|c\| \int_{0}^{t} d s\left\{\sup _{y \in D} h(t-s, y)^{\beta-1}\right\} \sum_{i=1}^{n} \prod_{i=1}^{n} T_{s} h_{t-s}\left(x_{i}\right) \\
& =n\|c\| \int_{0}^{t} d s\left\{\sup _{y \in D} h(s, y)^{\beta-1}\right\} \cdot \prod_{i=1}^{n} h\left(t, x_{i}\right),
\end{aligned}
$$

where we used that $T_{s} h_{t-s}(x)=h(t, x)\left({ }^{5}\right)$. Thus (3.1) is valid for $k=1$.

Suppose that (3.1) is valid for $k \geqq 1$. Then by (2.7) and the induction hypothesis, we have for $\boldsymbol{x}=\left(x_{1}, x_{2}, \ldots, x_{n}\right)$ and $m=n+\beta-1$

$$
\begin{aligned}
u_{k+1}(t, x)= & \int_{0}^{t} \int_{D^{m}} \Psi(x, d s d y) u_{k}(t-s, y) \\
\leqq & \|c\|^{k} \int_{0}^{t} \frac{\prod_{i=0}^{k-1}\{n+\beta-1+i(\beta-1)\}}{k !} \cdot\left\{\int_{0}^{t-s} d r \sup _{y \in D} h(r, y)^{\beta-1}\right\}^{k} \\
& \int_{D^{m}} \Psi(x, d s d y) \cdot \prod_{j=1}^{m} h\left(t-s, y_{j}\right) .
\end{aligned}
$$

By (2.5) this is equal to

$$
\begin{aligned}
& \|c\|^{k} \cdot \frac{\prod_{i=0}^{k-1}\{n+\beta-1+i(\beta-1)\}}{k !} \cdot \int_{0}^{t} d s\left\{\int_{0}^{t-s} d r \sup _{y \in D} h(r, y)^{\beta-1}\right\}^{k} \\
& \left.\qquad n\|c\|^{k+1} \cdot \frac{\prod_{i=0}^{n}\{n+\beta-1+i(\beta-1)\}}{k !} \cdot \int_{s}^{t} d s \cdot h_{t-s}^{\beta}\right)\left(x_{l}\right) \cdot \prod_{j \neq l} T_{s \in D} h(s, y)_{t-s}\left(x_{j}\right) \\
& \cdot\left\{\int_{0}^{s-1} d r \sup _{y \in D} h(r, y)^{\beta-1}\right\}^{k} \cdot \prod_{j=1}^{n} h\left(t, x_{j}\right) \\
& =\|c\|^{k+1} \cdot \frac{\prod_{i=0}^{k}\{n+i(\beta-1)\}}{(k+1) !} \cdot\left\{\int_{0}^{t} d s \sup _{y \in D} h(s, y)^{\beta-1}\right\}^{k+1} \cdot \prod_{j=1}^{n} h\left(t, x_{i}\right)\left({ }^{6}\right) .
\end{aligned}
$$

This proves (3.1) for $k+1$, completing the proof.

$\left({ }^{5}\right)$ We write sometimes $h_{t}(x)$ for $h(t, x)$.

$\left({ }^{6}\right)$ Note: $\int_{0}^{t} d F(s) F(s)^{k} / k !=F(t)^{k+1} /(k+1) !, F(0)=0$. 
Corollary 3.2. Let $u(t, x)$ be the probabilistic solution of $(2.1)$, then

$$
u(t, x) \leqq T_{t} f(x)\left\{1+\sum_{k=1}^{\infty} v_{k}(t)\right\}
$$

where

$$
v_{k}(t)=\frac{\prod_{i=0}^{k-1}\{1+i(\beta-1)\}}{k !}\left\{\|c\| \int_{0}^{t} \sup _{y \in D}\left(T_{s} f(y)\right)^{\beta-1} d s\right\}^{k}
$$

REMARK. When $T_{t} f(y)<1,(3.5)$ shows that larger $\beta$ provides better converging factor $\left(T_{t} f(y)\right)^{\beta-1}$. Therefore $\sum_{k=1}^{\infty} v_{k}(t)$ converges more easily for larger $\beta$.

THeOrem 3.3. For $f \in \boldsymbol{B}^{+}(D)$ satisfying

$$
(\beta-1)\|c\| \int_{0}^{\infty} \sup _{y \in D}\left(T_{t} f(y)\right)^{\beta-1} d t<1,
$$

there exists a global solution $u(t, x)$ of $(2.1)$.

Moreover there exists a constant $M>0$ such that

$$
u(t, x) \leqq M T_{t} f(x)\left({ }^{7}\right) .
$$

Proof. By (3.5) we have

$$
\sup _{t} \frac{v_{k+1}(t)}{v_{k}(t)} \leqq \frac{1+k(\beta-1)}{k+1} \cdot\|c\| \int_{0}^{\infty} \sup _{y \in D}\left(T_{t} f(y)\right)^{\beta-1} d t .
$$

Therefore (3.6) implies

$$
\sup _{t} \sum_{k=1}^{\infty} v_{k}(t)<\infty
$$

Thus the probabilistic solution actually provides a global solution. (3.7) follows from (3.4), completing the proof.

We shall give some applications of the preceding theorem.

Corollary 3.4. Suppose that the semigroup $T_{t}$ is transient in the following sense: For any open set $U \subset D$ with compact closure $\bar{U},(\bar{U} \neq D)$

$$
\int_{0}^{\infty} \sup _{x} T_{t}\left(I_{U}\right)(x) d t<\infty\left(^{8}\right) .
$$

If we assume $\beta \geqq 2$ and if $\delta>0$ is sufficiently small, then there exists a global solution $u(t, x)$ of $(2.1)$ for $f=\delta I_{U}$, and it satisfies (3.7).

$\left.{ }^{7}\right)$ In this case $u(t, x)$ is the unique bounded solution of (2.1), because $u^{\beta}$ satisfies locally Lipschitz's condition.

( $\left.{ }^{8}\right) I_{U}$ is the indicator of $U$. 
Proof. The assertion of this corollary is clear from

$$
\int_{0}^{\infty} \sup _{x}\left(T_{t} f(x)\right)^{\beta-1} d t \leqq \delta^{\beta-1} \int_{0}^{\infty} \sup _{x} T_{t} I_{U}(x) d t .
$$

THEOREM 3.5. Let $T_{t}$ be the semigroup of the d-dimensional symmetric stable process of index $\alpha(0<\alpha \leqq 2)$, i.e.

$$
\begin{aligned}
T_{t} f(x) & =\int_{R^{d}} p(t, x-y) f(y) d y, \\
e^{-t|z|^{\alpha}} & =\int_{R^{d}} e^{i(z, x)} p(t, x) d x\left(^{(}\right) .
\end{aligned}
$$

Let

$$
d(\beta-1) / \alpha>1,
$$

and $\gamma$ a positive number. Then there exists a positive number $\delta$ with the following property: If

$$
0 \leqq f(x) \leqq \delta p(\gamma, x),
$$

then there exists a global solution $u(t, x)$ of $(2.1)$ which satisfies

$$
0 \leqq u(t, x) \leqq M p(t+\gamma, x),
$$

for some positive constant $M$.

When $\alpha=2$, i.e., $T_{t}$ is the semigroup of the $d$-dimensional Brownian motion, this theorem was first proved by Fujita [2] by a different method.

Proof. If an initial data $f$ satisfies (3.11), we have

$$
T_{t} f(x) \leqq \delta p(t+\gamma, x) .
$$

Since

$$
p(t+\gamma, x)=(t+\gamma)^{-d / \alpha} p\left(1,(t+\gamma)^{-1 / \alpha} x\right)
$$

and

$$
p(1, y) \leqq p(1,0), \text { for } y \in R^{d},
$$

we have

$$
\begin{aligned}
\int_{0}^{\infty} \sup _{x}\left(T_{t} f(x)\right)^{\beta-1} d t & \leqq \delta^{\beta-1} p(1,0)^{\beta-1} \int_{0}^{\infty}(t+\gamma)^{-\alpha(\beta-1) / \alpha} d t \\
& =\delta^{\beta-1} p(1,0)^{\beta-1} \frac{\gamma^{1-\alpha(\beta-1) / \alpha}}{d(\beta-1) / \alpha-1} .
\end{aligned}
$$

Therefore if we take $\delta$ sufficiently small, (3.6) is satisfied. Hence the assertion of this theorem follows from Theorem 3.3.

$\left.{ }^{(}\right)|z|$ and $(z, x)$ denote norm and inner product, respectively. 
Remark. Put

$$
A=\sum_{i, j} a^{i j}(x) \frac{\partial^{2}}{\partial x^{i} \partial x^{j}}+\sum_{i} b^{i}(x) \frac{\partial}{\partial x^{i}},
$$

where $a^{i j}$ and $b^{i}$ are sufficiently smooth and subject to $\sum_{i, j} a^{i j}(x) \lambda_{i} \lambda_{j} \geqq \sum_{i} \lambda_{i}^{2}$ for all $x \in R^{d}$. Then it is known that the elementary solution $p(t, x, y)$ of $\partial u / \partial t=A u$ has the following upper bound:

$$
p(t, x, y) \leqq K t^{-d / 2} \exp \left(-a|x-y|^{2} / t\right),
$$

where $a$ and $K$ are certain positive constants. Therefore Theorem 3.5 is true when we take $\int p(t, x, y) d y f(y)$ as $T_{t} f(x)$, where we put $\alpha=2$.

4. Case 2. There exists no global solution. In order to obtain a criterion for existence of no global solution, we give a lower bound of $u_{k}(t, x)$.

LEMMA 4.1. Assume

$$
\inf _{x \in D} c(x)=c_{0}>0 \text {. }
$$

Then, for nonnegative $f \in \boldsymbol{B}(D)$ and $\boldsymbol{x}=\left(x_{1}, x_{2}, \ldots, x_{n}\right), u_{k}(t, x)$, which is defined by (2.7), has a lower bound

$$
\begin{aligned}
u_{k}(t, \boldsymbol{x}) \geqq & c_{0}^{k}\left\{\sum^{(k, n)} a_{k_{1} k_{2}} \cdots k_{n} h\left(t, x_{1}\right)^{k_{1}(\beta-1)} \cdot h\left(t, x_{2}\right)^{k_{2}(\beta-1)} \cdots h\left(t, x_{n}\right)^{k_{n}(\beta-1)}\right\} \\
& \cdot \prod_{j=1}^{n} h\left(t, x_{j}\right) \frac{t^{k}}{k !}
\end{aligned}
$$

where $k=1,2,3, \ldots, h(t, x)=T_{t} f(x)$, and $a_{k_{1} k_{2}} \ldots k_{n}$ are certain symmetric constants satisfying

$$
\sum^{(k, n)} a_{k_{1} k_{2}} \cdots k_{n}=n(n+\beta-1) \cdots(n+(k-1)(\beta-1)),
$$

where $\sum^{(k, n)}$ denotes the sum over all $\left(k_{1}, k_{2}, \ldots, k_{n}\right)$ satisfying $\sum_{i=1}^{n} k_{i}=k$.

Proof. We shall prove (4.2) by induction. Noting the following inequality which is justified by Jensen's inequality $\left({ }^{10}\right)$,

$$
T_{s}\left(h(t-s, \cdot)^{\beta}\right) \geqq\left\{T_{s}(h(t-s, \cdot))\right\}^{\beta}=h(t, x)^{\beta},
$$

we have by (2.7)

$$
\begin{aligned}
u_{1}(t, x) & =\int_{0}^{t} d s \sum_{l=1}^{n} T_{s}\left(c \cdot h_{t-s}^{\beta}\right)\left(x_{l}\right) \cdot \prod_{i \neq l} T_{s} h_{t-s}\left(x_{i}\right)\left({ }^{11}\right), \\
& \geqq c_{0} \int_{0}^{t} d s \sum_{l=1}^{n} h\left(t, x_{l}\right)^{\beta} \cdot \prod_{i \neq l} h\left(t, x_{i}\right),
\end{aligned}
$$

that is, (4.2) is verified for $k=1$, with $a_{00} \cdots 010 \cdots_{0}=1$.

( $\left.{ }^{10}\right) \beta \geqq 2$.

(11) We write $h_{t}(x)=h(t, x)$. 
Suppose that (4.2) is valid for $k \geqq 1$. By (2.7), the assumption, and (2.10), we have for $\boldsymbol{x}=\left(x_{1}, x_{2}, \ldots, x_{n}\right) \in D^{n}$ and $m=n-1+\beta$

$$
\begin{aligned}
& u_{k+1}(t, x)=\int_{0}^{t} \int_{D^{m}} \Psi(x, d s d y) u_{k}(t-s, y) \\
& \geqq c_{0}^{k} \int_{0}^{t} \int_{D^{m}} \Psi(x, d s d y)\left\{\sum^{(k, m)} a_{k_{1} k_{2}} \cdots k_{m} \prod_{i=1}^{m} h\left(t-s, y_{i}\right)^{k_{i}(\beta-1)+1}\right\} \cdot \frac{(t-s)^{k}}{k !} \\
& \geqq c_{0}^{k+1} \int_{0}^{t} d s \sum_{l=1}^{n} \sum^{(k, m)} a_{k_{1} k_{2}} \cdots k_{m} T_{s}\left(\prod^{\beta} h_{t-s}^{k_{l}(\beta-1)+1}\right)\left(x_{l}\right) \\
& \left.\prod_{j \neq l ; j=1}^{n} T_{s}\left(h_{t-s}^{k_{j}(\beta-1)+1}\right)\left(x_{j}\right) \cdot \frac{(t-s)^{k}}{k !}{ }^{(12}\right) \\
& \geqq c_{0}^{k+1} \sum_{l=1}^{n} \sum^{(k, m)} a_{k_{1} k_{2}} \cdots k_{m}\left\{h\left(t, x_{l}\right)^{)^{\beta}\left(k_{i}(\beta-1)+1\right)}\right\} \\
& \prod_{j \neq l: j=1}^{n} h\left(t, x_{j}\right)^{k_{j}(\beta-1)+1} \cdot{\frac{t^{k+1}}{(k+1) !}}^{\left({ }^{13}\right)},
\end{aligned}
$$

where we used (4.4) and performed the integration with respect to $s$ in the last step. The last line is equal to

$$
\begin{gathered}
c_{0}^{k+1} \sum_{l=1}^{n} \sum^{(k, m)} a_{k_{1} k_{2}} \cdots k_{m} h\left(t, x_{l}\right)^{\left(\sum^{\beta} k_{i}+1\right)(\beta-1)} \cdot \prod_{j \neq l}^{n} h\left(t, x_{j}\right)^{k_{j}(\beta-1)} \\
\cdot \prod_{j=1}^{n} h\left(t, x_{j}\right) \cdot \frac{t^{k+1}}{(k+1) !} \cdot
\end{gathered}
$$

If we introduce $k_{l}^{\prime}=\sum^{\beta} k_{i}+1$, this can be written as

$$
c_{0}^{k+1} \sum_{l=1}^{n} \sum^{(k, m)} a_{k_{1} k_{2}} \cdots k_{m} h\left(t, x_{l}\right)^{k_{l}^{\prime}(\beta-1)} \prod_{j \neq l} h\left(t, x_{j}\right)^{k_{j}(\beta-1)} \prod_{j=1}^{n} h\left(t, x_{j}\right) \cdot \frac{t^{k+1}}{(k+1) !}
$$

Consequently we have

$$
\begin{aligned}
u_{k+1}(t, x) \geqq & c_{0}^{k+1} \sum^{(k+1, n)} a_{k_{1} k_{2}}^{\prime} \cdots k_{n} h\left(t, x_{1}\right)^{k_{1}(\beta-1)} \cdot h\left(t, x_{2}\right)^{k_{2}(\beta-1)} \cdots h\left(t, x_{n}\right)^{k_{n}(\beta-1)} \\
& \cdot \prod_{j=1}^{n} h\left(t, x_{j}\right) \cdot \frac{t^{k+1}}{(k+1) !},
\end{aligned}
$$

where we put

$$
a_{k_{1} k_{2}}^{\prime} \cdots k_{n}=\sum_{l=1}^{n} \sum_{p_{l}+k_{n+1}+\cdots+k_{m}=k_{l}-1} a_{k_{1} \cdots p_{l} \cdots k_{m}} .
$$

This proves (4.2) for $k+1$. $a_{k_{1}}^{\prime} \ldots k_{n}$ are symmetric because so are $a_{k_{1}} \ldots k$. Moreover, since we have, by the induction hypothesis,

$$
\sum^{(k, m)} a_{k_{1} k_{2}} \cdots k_{m}=\{n+(\beta-1)\}\{n+2(\beta-1)\} \cdots\{n+k(\beta-1)\},
$$

(12) $\Pi^{\beta}$ denotes the product over $i=l, n+1, n+2, \ldots, m$.

(13) $\sum^{\beta}$ denotes the sum over $i=l, n+1, n+2, \ldots, m$. 
we have, noting (4.6),

$$
\sum^{(k+1, n)} a_{k_{1} k_{2}}^{\prime} \cdots k_{n}=n\{n+(\beta-1)\}\{n+2(\beta-1)\} \cdots\{n+k(\beta-1)\},
$$

which proves (4.3) for $k+1$, completing the proof.

COROllary 4.2. Let $u(t, x)$ be the probabilistic solution of $(2.1)$, then

$$
u(t, x) \geqq T_{t} f(x)\left\{1+\sum_{k=1}^{\infty} v_{k}(t, x)\right\}, \quad x \in D,
$$

where

$$
v_{k}(t, x)=\frac{1}{k !} \prod_{j=0}^{k-1}\{1+j(\beta-1)\}\left\{c_{0} t\left(T_{t} f(x)\right)^{\beta-1}\right\}^{k} .
$$

THEOREM 4.3. For $f \in B^{+}(D)$ satisfying, for some $x_{0} \in D$ and $t_{0}>0$,

$$
(\beta-1) c_{0} t_{0}\left(T_{t_{0}} f\left(x_{0}\right)\right)^{\beta-1}>1\left({ }^{14}\right),
$$

all solution $u(t, x)$ of the equation (2.1) blows up at a point in a finite time interval (i.e. no global solution exists).

Proof. By [a.3] the probabilistic solution $u(t, x)$ is the minimal solution of (2.1). Therefore it is sufficient to consider this solution $u(t, x)$. Assume that $u(t, x)$ does not blow up all $t>0$. Then $u(t, x)$ satisfies (4.7). We have, however, for sufficiently large $k$

$$
\frac{v_{k+1}\left(t_{0}, x_{0}\right)}{v_{k}\left(t_{0}, x_{0}\right)}=\frac{1+k(\beta-1)}{k+1} c_{0} t_{0}\left(T_{t_{0}} f\left(x_{0}\right)\right)^{\beta-1}>1,
$$

which contradicts the assumption.

We shall give some applications of the above theorem.

Corollary 4.4( $\left(^{15}\right)$. Let $D$ be a bounded domain in $R^{d}$ and let $T_{t}$ be the semigroup of an A-diffusion on $D$ with absorbing boundary $\left({ }^{16}\right)$. If the initial data $f \geqq 0$ takes sufficiently large values on an open set with positive Lebesgue measure, then the solution $u(t, x)$ of $(2.1)$ blows up in a finite time interval ${ }^{(17)}$.

REMARK. In the above corollary, $A$-diffusion with absorbing boundary is a process on $\bar{D}=D \cup\{\delta\}$ (one-point compactification of $D$ ) with $\delta$ as the terminal point. We always assume $f(\delta)=0$ for $f \in \boldsymbol{B}(D)$.

(14) $c_{0}=\inf _{x \in D}$ inf $c(x)>0$.

${ }^{(15)}$ A different proof of this theorem is given in S. Ito [6].

$\left.{ }^{(18}\right)$ This is the process with transition probability $p(t, x, y) d y$, where $p(t, x, y)$ is the elementary solution of $\partial u / \partial t=A u, u \mid \partial_{D}=0, A=a^{i j}(x)\left(\partial^{2} / \partial x^{i} \partial x^{j}\right)+b^{t}(x) \partial / \partial x^{i}$.

$\left({ }^{17}\right)$ We assume inf $x \in D C(x)=c_{0}>0$. 
THEOREM 4.5( $\left.{ }^{18}\right)$. Let $T_{t}$ be the semigroup of the d-dimensional symmetric stable process with index $\alpha(0<\alpha \leqq 2)$. Let

$$
0<d(\beta-1) / \alpha<1 .
$$

Then for any nonnegative measurable function $f$ on $R^{d}$ which has strictly positive values in an open set with positive Lebesgue measure, all solution $u(t, x)$ of $(2.1)\left({ }^{19}\right)$ blows up in a finite time interval, i.e., (2.1) has no global solution.

Proof. First of all we note that we have, if $t \geqq 1, T_{t} f(x) \geqq t^{-d / \alpha} T_{1} f(x)$. On the other hand there exists $x_{0} \in R^{d}$ such that $0<T_{1} f\left(x_{0}\right)$ by the assumption. Therefore under the condition (4.10), we have, if $t$ is sufficiently large,

$$
(\beta-1) c_{0} t\left(T_{t} f\left(x_{0}\right)\right)^{\beta-1}=(\beta-1) c_{0} t^{1-\alpha(\beta-1) / \alpha}\left(T_{1} f\left(x_{0}\right)\right)^{\beta-1}>1 .
$$

Hence $u(t, x)$ blows up in a finite time interval by Theorem 4.3. This completes the proof.

ACKnowledgement. The authors wish to express their thanks to Professor N. Ikeda who had discussions with them and gave advice in preparing this paper, and to Professor H. Kesten who read their original manuscript and suggested some corrections.

\section{REFERENCES}

1. E. B. Dynkin, Markov processes, Springer, New York, 1965.

2. H. Fujita, On the blowing up of solutions of the Cauchy problem for $u_{t}=\Delta u+u^{1+\alpha}$, J. Fac. Sci. Univ. Tokyo 13 (1966), 109-124.

3. N. Ikeda, M. Nagasawa and S. Watanabe, Branching Markov processes, J. Math. Kyoto Univ. 8 (1968). Abstracts, Proc. Japan Acad. 41 (1965), 816-821 ; 42 (1966), 252-257, 370-375, 380-384, 719-724, 1016-1021, 1022-1026.

4. - Foundation of branching Markov processes, Seminar on Probability Vol. 23, 1966. (Japanese)

5. K. Ito and H. P. McKean, Jr., Diffusion processes and their sample paths, Springer, New York, 1965.

6. S. Ito, On the blowing up of solutions of semi-linear parabolic equations. Bull. Math. Soc. Japan (Sûgaku) 18 (1966) 44-47. (Japanese)

7. M. Nagasawa, On construction of branching Markov process with age and sign, Kōdai Math. Sem. Rep. 20 (1968), 469-508.

8. T. Sirao, On signed branching Markov processes with age, Nagoya Math. J. 32 (1968), 155-225.

TOKYo INSTITUTE OF TECHNOLOGY,

TOKYO, JAPAN

CORNELl UNIVERSITY,

IthaCA, New York

NAGOYA UNIVERSITY,

NAGOYA, JAPAN

${ }^{(18)}$ This result was first proved by Fujita [2] in the case $\alpha=2$, i.e., for Brownian motion by a different method.

(19) We assume $\inf _{x \in R^{d}} c(x)=c_{0}>0$. 